\title{
Les Organisations Non-Gouvernementales (ONGs) de défense des droits de l'homme au Burundi : promouvoir la revendication judiciaire des droits.
}

\author{
Par Nestor Nkurunziza ${ }^{1}$
}

La fin de la Guerre froide avec le triomphe du libéralisme occidental marqua le début du processus de diffusion des valeurs fondatrices de la démocratie libérale à travers le monde. Au sein du système onusien, le domaine des droits de l'homme et, du moins si on s'en tient à l'adoption et la diffusion des standards internationaux, reste une des grandes réalisations dans cette direction ${ }^{2}$. Sur le plan normatif, un arsenal juridique international des droits de l'homme a été élaboré avec l'adoption, en 1966, des deux pactes internationaux, respectivement le Pacte International relatif aux Droits Civils et Politiques (PIDCP) ${ }^{3}$ ainsi que celui sur les droits économiques, sociaux et culturels (PIDESC) ${ }^{4}$ marquant le début d'une ère de prolifération de textes liés aux droits de l'homme ${ }^{5}$. A son tour, l'adhésion par les Etats à ces textes s'est à son tour universalisée. Plus de quatre vingt pourcent des pays membres de l'ONU sont aujourd'hui parties aux deux pactes qui revêtent un caractère contraignant ${ }^{6}$. Corollairement à la mise en place du cadre normatif de promotion et de protection des

1 Licence en Droit, M. Phil. International Peace Studies (TCD, Dublin, République d'Irlande), DESS en Droit de l'Homme et Résolution Pacifique des Conflits (Chaire Unesco en Education à la Paix et Résolution Pacifique des Conflits/Université du Burundi)Maître-assistant attaché à la Faculté de Droit, Université du Burundi.

2 Dès sa création en 1945, l'Organisation des Nations Unies (ONU) avait à son agenda l'adoption des standards internationaux des droits de l'homme quoique la Guerre froide freina le processus. Selon Eléonore Roosevelt, la Déclaration Universelle des Droits de l'Homme (DUDH, 1948) devait servir comme « ...l'idéal commun à atteindre par tous les peuples et toutes les nations », citée dans KAREN A. MINGST and MARGARET P. KARNS (2007) The United Nations in the 21st Century. Oxford: Westview Press, p. 169. Texte original en Anglais: "...to serve as a common standard of achievement for all people and nations." Traduction empruntée sur le site web du Haut Commissariat aux Droits de l'Homme des Nations en suivant le lien: http://www.ohchr.org/FR/ProfessionalInt erest/Pages/InternationalLaw.aspx, consulté le 27 mars 2013.

3 Nations Unies, Recueil des Traités, vol. 999, p. 171 et vol. 1057, p. 407.

4 Nations Unies, Recueil des Traités, vol. 993, p. 3. Ensemble avec la DUDH, le PIDESC, le PIDCP ainsi que ses deux protocoles forment la charte internationale des droits de l'homme. Accessible sur le site web du Haut Commissariat des Nations Unies aux Droits de l'Homme en suivant le lien : http://www2.ohchr.org/french/law/index.htm\#core, consulté le 12/08/2013.

5 Voir Nations Unies, Collection des Traités pour la liste complète des différents instruments. Accessible en suivant le lien : $\mathrm{http}$ ://treaties.un.org/Pages/Treaties.aspx id=4\&subid=A\&lang=fr\&clang= _fr, consulté le 28 mars 2013.

6 Sur les 193 Etats membres de l'ONU 167 sont parties au PICDP; 160 au PIDESC. Les Etats-Unis d'Amérique sont parties au premier, ont signé mais pas ratifié le second. Voir ibid. 
droits de l'homme, des mécanismes de contrôle de leur mise en application ont été instaurés et progressivement renforcés au sein du système onusien ${ }^{7}$.

En vue de compléter les mécanismes onusiens, des instruments et des mécanismes régionaux ont été créés ${ }^{8}$. A côté de ces cadres supranationaux, l'étape la plus marquante vers la « mondialisation » des standards internationaux des droits de l'homme, reste leur incorporation systématique dans la quasi-totalité des législations nationales dans la plupart des Etats modernes.

7 Il existe neuf organes spécifiquement chargés de surveiller la mise en œuvre des principaux traités des droits de l'homme à l'échelle onusienne : le Comité des droits de l'homme (CCPR), le Comité des droits économiques, sociaux et culturels (CERD), le Comité pour l'élimination de la discrimination à l'égard des femmes (CEDAW), le Comité contre la torture (CAT), le Sous Comité pour la prévention de la torture (SPT), le Comité des droits de l'enfant (CRC), le Comité des travailleurs migrants (CMW), le Comité des droits des personnes handicapées (CRPD)ainsi que le Comité des disparitions forcées (CED). le Conseil des droits de l'homme, Examen périodique universel, la Commission des droits de l'homme ainsi les Procédures spéciales assumées par le Conseil des droits de l'homme sont en revanche des mécanismes créés en vertu de la Charte des Nations Unies.

8 En ce qui concerne l'Afrique, la Charte Africaine des Droits de l'Homme et des Peuples (ici dénommée la Charte africaine ou la Charte) fut adoptée le juin 1981 au cours de la dix huitième Conférence de Chefs d'Etats et de Gouvernements membres de l'Organisation de l'Unité Africaine (OUA, actuelle Union Africaine, UA), entrée en vigueur 21 octobre 1986, O.A.U. Doc. CAB/LEG/ 67/3 rev. 5, publiée dans 21 I.L.M. 58 (1982). Le mécanisme institutionnel en charge de sa mise en application comprend une Commission Africaine des Droits de l'Homme (ci après la Commission africaine ou la Commission) et une Cour Africaine des Droits de l'Homme et des Peuples (ci après la Cour Africaine ou la Cour). Chargée notamment « de promouvoir les droits de l'homme et d'assurer leur protection en Afrique » en vertu de l'article 30 de la Charte, la Commission a été mise en place en 1987. Elle est restée le seul organe de contrôle de la mise application de la Charte africaine jusqu'à l'adoption, en 1998, d'un Protocole relatif à la Charte africaine portant création d'une Cour Africaine des Droits de l'Homme et des Peuples (Protocole portant création de la Cour ou le Protocole), entré en vigueur en janvier 2004, O.A.U.Doc. OAU/LEG/EXP/AFCHPR/PROT (III). La Cour fut effectivement mise en place en 2006 et « complète les fonctions de protection que la Charte a conférées à la Commission. ", art. 2 du protocole. Au niveau européen, la Convention Européenne pour la sauvegarde des Droits et Libertés Fondamentales a été adoptée en 1950 et entrée en vigueur en 1953. Pour plus de discussion sur le contenu normatif de la Convention et ses protocoles ainsi que le système institutionnel mis en place avec un commentaire sur des affaires emblématiques, voy. Henry J. Steiner et al. (2008), pp. 933-1013; le système interaméricain est régi à la fois par le texte initial non contraignant de la Déclaration Américaine des Droits et Devoirs de l'Homme (1948) ainsi que la Convention Américaine des Droits de l'Homme (1969); voy. Henry J. Steiner et al. (2008, pp. 1020-1062 pour un commentaire général sur les aspects institutionnels du système ainsi que le contenu normatif des deux instruments applicables. Pour des travaux spécialisés, voy. Notamment Medina, C. (1990) The Inter-American Commission on Human Rights and the Inter-American Court of Human Rights: Reflections on a Joint Venture, Human Rights Quarterly, Vol. 12, 4, pp. 439-464; Harris, D. (1998) Regional Protection of Human Rights : The Inter-American Achievement. In D. Harris and S. Livingstone (eds.), The Inter-American System of Human Rights.Oxford: Clarendon Press, Chap.1; FARER, T. The Rise of the Inter-American Human Rights Regime: No Longer a Unicorn, not yet an Ox. In Id., Chap. 32; voy. Henry J. Steiner et al. (2008)

pp. 1042-1062 pour une jurisprudence sélectionnée. 
Le processus de démocratisation des instituions -accompagné de la mondialisation des droits de l'homme- n'a pour autant pas conduit à l'émergence d'une «culture globale $»^{9} \mathrm{de}$ respect de la dignité humaine au cœur même de l'idéal des droits de l'homme. Cette observation est particulièrement valable en ce qui concerne la situation des droits de l'homme sur le contient africain. L'adhésion de la plupart des Etats africains aux principaux des instruments des droits de l'homme ne s'est pas accompagnée d'une amélioration des conditions de vie de leurs populations. D'une part et comme par un paradoxe, les mêmes Etats se sont livrés à des violations graves et massives des mêmes droits qu'ils s'étaient engagés à promouvoir et garantir ${ }^{10}$. D'autre part et plus préoccupant encore, la libéralisation des sociétés et des institutions africaines n'a pas mené à une culture de revendication, par les populations, des libertés et droits fondamentaux qui leur sont pourtant reconnus par les lois fondamentales de leurs pays.

Le Burundi peut être considéré comme un cas d'illustration du phénomène. Il a ratifié ou adhéré à la plupart des instruments internationaux et régionaux des droits de l'homme. Les normes internationales des droits de l'homme ainsi garanties font partie intégrante du droit positif burundais et sont directement applicable devant le juge national, ont rang de normes constitutionnelles primant ainsi toutes règles de valeur infra-constitutionnelle. La Cour constitutionnelle est la plus haute juridiction compétente pour veiller au respect de ces standards internationaux. Malgré ce cadre normatif et institutionnel plutôt favorable, la protection juridictionnelle des droits de l'homme n'est pas encore entrée dans la pratique judiciaire burundaise ${ }^{11}$.

9 Pour plus de discussion sur une perspective constructiviste sur les normes et les instituions internationales, voy. particulièrement Ruggie, J. Gerard (1998) Constructing the World Polity: Essays on International Institutionalization, London: Routledge; Finnemore, M. (1996) "Norms, culture, and world politics: insight from sociology's institutionalism", International Organization 50, 2, Spring, pp. 325-347. La thèse constructiviste dans les politiques internationales repose sur le postulat selon lequel grâce à des acteurs variés qui ne comprennent pas uniquement les Etats mais également les organisations internationales, l'ONU en tête, on évoluerait vers une culture globale, une mondialisation des valeurs. La prolifération ainsi que l'adhésion quasi générale aux normes internationales des droits de l'homme sont citées comme des exemples illustratifs du phénomène. Les réalistes de leur côté, mettent un accent sur le rôle central des intérêts et des pratiques plutôt étatiques. En ce qui concerne l'adhésion aux instruments des droits de l'homme, en particulier, certains Etats le feraient pour des raisons tout autres que le souci d'une garantie de leur respect. En ce sens, des Etats seraient « contraints » d'adhérer à des normes et des valeurs auxquels ils ne sont pas effectivement acquis. Dans ce sens, voy. Par exemple, Ikenberry, G. John (2001) After Victory : Institutions, Strategic Restraint, and the Rebuilding of Order after Major Wars. Princeton, NJ: Princeton University Press. Egalement cited ans Weiss, Thomas G.and Daws, S (2007) The Oxford Handbook on the United Nations. Oxford University Press, p. 44.

10 Le professeur Mutua donne l'exemple du Zaïre (ex République Démocratique du Congo) de Mobutu qui avait ratifié la plupart des principaux instruments des droits de l'homme alors qu'il menait parallèlement une politique officielle de violation des mêmes droits. Voy. Mutua, M. "The ideology of human rights", 36 Va.J. Int'l L.: 589 1995-1996, pp.599-600; 641.

11 Dans ce sens voy. Cistac, G. (2008) Code de justice administrative du Burundi, commenté et annoté. Bujumbura : La Licorne, p.121; également cité dans Ntahiraja, B. et Nkurunziza, N. (2010) 
Cet article épouse et part de l'idée selon laquelle la solution durable aux violations des droits de l'homme réside dans la volonté et la capacité des populations [victimes] ${ }^{12} \mathrm{de}$ s'opposer à l'oppression et aux violations de leurs droits [par les Etats] ${ }^{13}$. Comme l'indique le sous-titre si évocateur de l'ouvrage Human Rights under African Constitutions : Realizing the Promise by Ourselves par An-Na'im, Abdullahi et ses collègues, les droits de l'homme ne deviendront réalité sur le continent africain qu'à partir du moment où les populations (victimes, individus) se seront imprégnées de la lutte pour le respect des droits de l'homme $^{14}$. Avec ces idées à l'esprit, l'argument de cet article est que les Organisations Non-Gouvernementales (ONGs), citoyens organisés ${ }^{15}$, portent la responsabilité première de ce combat; elles ont le devoir de promouvoir l'éclosion et le développement de cette culture de résistance au sein des masses. Dans ce sens et dans le cas du Burundi, les défenseurs des droits de l'homme doivent intégrer la revendication judiciaire des droits qui fait actuellement défaut dans leurs programmes et stratégies d'action. Bien plus et plus important, elles doivent œuvrer à l'émancipation des masses afin que celles-ci puissent prendre dans leurs mains la revendication de leurs droits. Nous soutenons que les économiques, sociaux et culturels constituent un outil particulièrement efficace dans ce processus d'émancipation. A cette fin et sur le plan des droits de l'homme, les ONGs devraient accorder davantage de considération aux droits économiques, sociaux et culturels qu'il en est actuellement le cas. Il conviendra d'explorer brièvement le cadre normatif des droits de l'homme au Burundi avant de développer.

'L'Etat face aux droits sociaux de ses citoyens: cas du droit à la santé au Burundi. ', Rule of Law Program For Sub-Saharan Africa, Librairie Africaine d'Etudes Juridiques, Vol. 7: p.20-45;voy. dans le meme sensVandenginste, S. (2011), supra, n.10, p.289 citant Ndayikengurukiye, M. (2005) The international human rights law as a source of law in the Burundian judicial system, Master Dissertation, Makerere University, Kampala. Vandenginste tire cette conclusion également sur base d'une série d'interviews menée auprès des praticiens du droit.

12 Makau Mutua analyse le combat pour le respect des droits de l'homme selon la thèse libérale sous forme d'une métaphore mettant aux prises l'individu (victime potentielle) qu'il faut protéger contre son prédateur (l'Etat oppresseur). Voy. Mutua, M. (2001) 'Savages, Victims, and Saviors : The Metaphor of Human Rights', Harvard International Law journal, 42, pp. 201-245.

13 " (...) the ultimate success of this approach is contingent on the willingness and ability of the people themselves to oppose oppression and violation of their rights(...)" Abdullahi, A. (2003) Expanding Legal Protection of Human Rights in African Contexts in AnP:|Wissenschaft $\mid$ NestlerAsholt_0148-3\4. Umbruch-Na'im, Abdullahi (ed.), Human Rights Under African Constitutions, Realizing the Promise for Ourselves. Philadelphia: University of Pennsylvania Press, p.4.

14 "It is therefore clear that the beneficiaries of human rights standards themselves must bear primary responsibility for protecting their own rights (...)". Id., p.7.

15 Les activistes de la société préfèrent généralement se présenter comme de simples citoyens organisés et non des représentants des masses traduisant ainsi l'idée d'une communauté de sort avec ces dernières. 
I. Les obligations du Burundi en vertu du droit international des droits de l'homme et statut de la norme internationale en droit positif burundais

Le Burundi a ratifié ou adhéré aux principaux instruments internationaux et régionaux de protection des droits de l'homme dont découlent une série d'obligations internationales. Nous passons brièvement en revue ces obligations à caractère général avant de parler du statut des normes internationales des droits de l'homme en droit positif burundais. Un tel commentaire sur les opportunités qui s'offrent aux justiciables pour invoquer les normes internationales des droits de l'homme devant les instances nationales constituera une introduction utile à la deuxième section de l'article. Celui-ci nous permettra, en effet, de vérifier si les titulaires des droits exploitent effectivement les voies de revendication qui s'offrent à eux et de formuler les recommandations conséquentes.

Le tableau ci après reprend la liste des principaux instruments pertinents dans le cas du Burundi :

\begin{tabular}{|c|c|c|c|}
\hline Instrument (date d'adoption) & $\begin{array}{l}\text { Date de } \\
\text { signature }\end{array}$ & $\begin{array}{l}\text { Date de ratifica- } \\
\text { tion (ou } \\
\text { adhésion) }\end{array}$ & $\begin{array}{l}\text { Entrée en } \\
\text { vigueur }\end{array}$ \\
\hline $\begin{array}{l}\text { 1. Convention internationale sur l'élimination de } \\
\text { toutes les formes de discrimination raciale } \\
(\mathrm{CDR})(21 \text { décembre } 1965)\end{array}$ & $01 / 02 / 1967$ & $27 / 10 / 1977$ & $26 / 11 / 1977$ \\
\hline $\begin{array}{l}\text { 2. Charte Africaine des Droits de l'Homme et } \\
\text { des Peuples (CADHP) }(27 / 06 / 1981) \\
\text { Protocole relatif à la Charte africaine des droits } \\
\text { de l'Homme et des peuples portant création } \\
\text { d'une Cour africaine des droits de l'Homme et } \\
\text { des peuples }\end{array}$ & $09 / 06 / 1998$ & $\begin{array}{l}28 / 07 / 1989 \\
02 / 04 / 2003\end{array}$ & $\begin{array}{l}30 / 11 / 1989 \\
25 / 01 / 2004\end{array}$ \\
\hline $\begin{array}{l}\text { 3. Pacte international relatif aux droits civils et } \\
\text { politiques (PIDCP) (16 décembre 1966) }\end{array}$ & & $09 / 05 / 1990$ & $09 / 08 / 1990$ \\
\hline $\begin{array}{l}\text { 4. Pacte international relatif aux droits écono- } \\
\text { miques, sociaux et culturels (PIDESC) (16 dé- } \\
\text { cembre 1966) }\end{array}$ & & $09 / 05 / 1990$ & \\
\hline $\begin{array}{l}\text { 5. Convention relative aux droits de l'enfant } \\
\text { (20 novembre 1989) }\end{array}$ & $08 / 05 / 1990$ & $19 / 10 / 1990$ & $18 / 11 / 1990$ \\
\hline $\begin{array}{l}\text { 6. Convention sur l'élimination de toutes les } \\
\text { formes de discrimination à l'égard des } \\
\text { femmes(CEDF) (18 décembre 1979) }\end{array}$ & $17 / 071980$ & $08 / 01 / 1992$ & $07 / 02 / 1992$ \\
\hline $\begin{array}{l}\text { 7. Convention contre la torture et autres peines } \\
\text { ou traitements cruels, inhumains ou dégra- } \\
\text { dants (10 décembre 1984) }\end{array}$ & & $18 / 02 / 1993$ & $20 / 03 / 1993$ \\
\hline $\begin{array}{l}\text { 8. Charte Africaine des droits et du bien-être de } \\
\text { l'enfant }\end{array}$ & & $28 / 06 / 2004$ & $23 / 09 / 2004$ \\
\hline
\end{tabular}


Les Organisations Non-Gouvernementales (ONGs) de défense des droits de l'homme au Burundi : promouvoir la revendication judiciaire des droits.

Obligations générales en vertu du droit international des droits de l'homme

Par la ratification ou l'adhésion aux instruments des droits de l'homme, l'Etat partie s'engage à une série d'obligations. Ces obligations sont communes aux instruments des droits de l'homme tant internationaux que régionaux ${ }^{16}$.La Commission Africaine des Droits de l'Homme et des Peuples (la Commission Africaine), dans sa décision célèbre, SERAC c. Nigéria, a tenu à le rappeler dans ces termes,

Les idées acceptées au plan international concernant les diverses obligations créées par les droits de l'homme indiquent que tous les droits, civils et politiques, sociaux et économiques, créent au moins quatre niveaux d'obligations pour un Etat qui s'engage à adopter un régime de droits, notamment le devoir de respecter, de protéger, de promouvoir et de réaliser ces droits ${ }^{17}$.

«Ces obligations », poursuit la Commission, « s'appliquent universellement à tous les droits et imposent une combinaison de devoirs négatifs et positifs $»^{18}$. Chacune de ces obligations implique à la fois des prestations et des abstentions ${ }^{19}$.En vertu de l'obligation de respecter, l'Etat doit s'abstenir de porter atteinte, du fait de ses agents ou des personnes agissant sous ses ordres, aux droits reconnus ${ }^{20}$. Il doit également s'abstenir de toute restriction non autorisée à l'exercice des droits ${ }^{21}$.

L'obligation de protéger impose à l'Etat le devoir de prévenir d'éventuelles violations commises par des particuliers, « [...] personnes privées, physiques ou morales, qui entraveraient l'exercice des droits énoncés $[. ..] »^{22}$ et de garantir des voies de recours aux victimes de telles violations. Comme le Comité des Droits de l'Homme des Nations Unies(le Comité des droits de l'homme le précise,

[...]il peut arriver qu'un manquement à l'obligation [...] de garantir les droits reconnus [...] se traduise par une violation de ces droits par un État partie si celui-ci tolère de tels actes ou s'abstient de prendre des mesures appropriées ou d'exercer la

16 Par ex. PIDCP et PIESC Art. 2., para. 1-2; CADHP, art. 25.

17 Communication 155/96, Social and Economic Rights Action Center (SERAC) and Center for Economic and Social Rights (CESR) c. Nigéria, CADHP, 15ème Rapport d'activités: 2001 - 2002 (ici SERAC et Autre c. Nigéria), para. 44.

18 SERAC et Autre c. Nigéria, ibid. Dans le meme sens, Comité des Droits de l'Homme, Observation Générale $\mathrm{N}^{\circ} 31$, La nature de l'obligationjuridique générale imposée aux États parties au Pacte, CCPR/C/21/Rev. 1/Add.13, 26 mai 2004, para.6 (ici Observation générale n 31).

19 Observation générale $\mathrm{n}^{\circ} 31$, para. 6.

20 Pour une analyse plus détaillée, voy. Drzewicki, K. (1999) 'Internationalization of Human Rights and theirJuridization'. In Raija Hanski and MarkuSuksi (Eds.), Revised Edition, An Introduction to the International Protection of Human Rights: A Textbook, p. 31; également cité dans SERAC et Autre c. Nigéria, para. 45; au niveau de l'ONU, voy. Observation Générale n 31, supra, ns. 16;18, paras.6-8.

21 Ibid.

22 Observation générale $\mathrm{n}^{\circ} 31$,supra, ns. 16; 18, para. 8. 
diligence nécessaire pour prévenir et punir de tels actes commis par des personnes privées, physiques ou morales, enquêter à leur sujet ou réparer le préjudice qui en résulte en sorte que lesdits actes sont imputables à l'État partie concerné23.

Enfin, les Etats sont tenus de promouvoir et de réaliser les droits énoncés dans les instruments auxquels ils sont parties. Les mesures tendant vers la réalisation des droits peuvent être d'ordre législatif, judiciaire, administratif, éducatif ou autres ${ }^{24}$. Les Etats doivent, selon les termes du Comité des droits de l'homme, « (...) orienter leurs systèmes vers la réalisation effective des droits $»^{25}$. En ce sens, le Comité, a estimé que, si le PIDCP n'exige pas nécessairement que le Pacte soit directement applicable par voie d'incorporation dans le droit interne,

[...]les droits garantis par le Pacte sont susceptibles d'être mieux protégés dans les États où le Pacte fait partie de l'ordre juridique interne automatiquement ou par voie d'incorporation expresse ${ }^{26}$.

En distinguant les systèmes dans lesquels les droits garantis sont applicables automatiquement de ceux où il faut une procédure d' « incorporation expresse », le texte du comité semble faire allusion aux systèmes moniste d'une part et dualiste, d'autre part. Ces observations nous mènent une analyse du statut des normes internationales des droits de l'homme en droit burundais.

Statut de la norme internationale des droits de l'homme en droit burundais Applicabilité directe

Les modalités d'application d'une norme du droit international en droit interne diffèrent selon que l'on se trouve dans un système moniste ou dualiste. Dans la première hypothèse, la norme internationale contenue dans un instrument international ratifié pourra être directement invoquée devant le juge national alors qu'il faudrait une procédure spéciale de transposition (généralement par l'adoption d'une loi dite de réception) de la norme dans le cadre normatif national pour qu'elle soit applicable par les juridictions nationales dans la seconde.

Une interprétation de l'article 292 de la Constitution de la République du Burundi permet de conclure que le Burundi adhère plutôt au système moniste ${ }^{27}$ rendant ainsi les normes

23 Drzewicki, K. (1999), n. 19, p.31.

24 Observation générale n³1,para. 7.

25 SERACet Autre c. Nigéria, supra, n.16;17,para. 47.

26 Observation générale $\mathrm{n}^{\circ} 31, \mathrm{n} .85$, para. 13.

27 Quoiqu'en dehors d'une procédure judiciaire, la Cour Suprême du Burundi a confirmé que le Burundi a adhérait plutôt à un système moniste. Cette réponse a été donnée en réponse à une question dans ce sens par l'Association des Hautes Juridictions de cassation des pays ayant en partage l'usage du français (AHJUCAF). L'enquête avait été menée sur base d'un questionnaire intitulé « Les Conventions et la jurisprudence devant le juge national » dont les résultats ont été présentés 
Les Organisations Non-Gouvernementales (ONGs) de défense des droits de l'homme au Burundi : promouvoir la revendication judiciaire des droits.

internationales, directement applicables, devant les juridictions burundaises. Aux termes de cet article,

Les traités ne prennent effet qu'après avoir été régulièrement ratifiés et sous réserve de leur application par l'autre partie pour les traités bilatéraux et de la réalisation des conditions de mise en vigueur prévues par eux pour les traités multilatéraux ${ }^{28}$.

La ratification, quant à elle, relève des compétences du président de la République et exceptionnellement du Parlement en ce qui concerne les traités portant sur certaines matières déterminées ${ }^{29}$. Vandenginste fait remarquer que la nature moniste du système burundais se trouve tempéré du fait que la ratification de certaines catégories de traités relève ainsi de la compétence du législateur ${ }^{30}$. Ce tempérament n'entraîne pas pour autant une perte du caractère moniste puisque, comme cet auteur le note lui-même, la ratification, même par le parlement, consiste le plus souvent, en un article unique ${ }^{31}$ consacrant ainsi l'entrée de la Convention dans l'ordre interne. Or, dans un système dualiste, l'acte de " domestication » du traité consisterait normalement en l'adoption d'une loi (loi de réception) par le législateur national reprenant intégralement le contenu du traité, disposition par disposition, le cas échéant, puisque dans une conception dualiste, le droit interne et le droit international constituent deux blocs séparés. Dans un système moniste, par contre, les deux sources du droit appartiennent à la même catégorie ${ }^{32}$.

Comme par ailleurs les instruments relatifs aux droits de l'homme se retrouvent généralement dans la catégorie de "traités multilatéraux », une interprétation a contrario du texte de l'article 292 laisse entendre que deux conditions suffisent pour qu'ils prennent effet : la ratification d'une part et, d'autre par, l'entrée en vigueur de l'instrument en question.

Le principe de l'applicabilité directe du droit international a, du reste, été confirmé à maintes reprises aussi bien à l'occasion de déclarations officielles que sur le plan jurispru-

à l'occasion du premier congrès général de l'AHJUCAF en 2004. Cité dans Vandenginste, S. (2011), supra, n.10, p.285 (note 789.).

28 Loi N ${ }^{\circ} 1 / 010$ du 18 mars 2005 portant promulgation de la Constitution de la République du Burundi. In B.O.B 2005, $n^{\circ} 3$ Ter, pp. 1-35.

29 Articles 289 et 290, ibid. Art. 289 : « Le Président de la République a la haute direction des négociations internationales. Il signe et ratifie les traités et accords internationaux »; art. 290 : « les traités de paix et les traités de commerce qui engagent les finances de l'Etat, ceux qui modifient les dispositions de nature législative ainsi que ceux qui sont relatifs à l'état des personnes ne peuvent être ratifiés qu'en vertu d'une loi ».

30 Vandenginste, S. (2011), p. 285.

31 Vandenginste, S. (2011), ibid.

32 Se référant à Hans Kelsen comme l'auteur porte flambeau sur la théorie du monisme, Antonio Cassese définit la conception moniste comme celle qui « maintient l'unité des différents systèmes juridiques avec une primauté du droit international sur les lois et règlements. ", " maintaining the unity of various legal systems and the primacy of international law". Voy. Cassese, A. (2005) International law. Oxford: Oxford University Press, p.215. Cité dans Vandenginste, S. (2011), supra, n.94, p. 285 (note 790.). 
dentiel. Officiellement, le document de base soumis par l'Etat du Burundi « conformément aux directives unifiées concernant la première partie des rapports présentés par les Etats parties en vertu des divers instruments relatifs aux droits de l'homme (HRI/1991/1) », le confirme ainsi,

Les dispositions des divers instruments relatifs aux droits de l'homme peuvent être invoquées devant les instances judiciaires ou les autorités administratives et appliquées directement par celles- $i^{33}$.

La Cour Constitutionnelle est compétente pour interpréter la Constitution et est garante de son respect par les autorités administratives et judiciaires ${ }^{34}$. Dans ce sens, un attendu de l'arrêt $R C C B 8$ de la Cour Constitutionnelle se lit ainsi,

Attendu en effet que l'article 10 de la Déclaration Universelle des Droits de l'Homme et l'article 14 alinéa premier du Pacte international relatif aux droits civils et politiques, applicables par l'effet de l'article $10^{35}$ de la Constitution consacrent $[\ldots]^{36}$

Cet attendu qui applique des articles respectivement de la DUDH et du PIDCP en passant par une disposition constitutionnelle (art. 10) ne devrait pas laisser sous-entendre que les mêmes normes internationales seraient inopérantes en l'absence d'une disposition qui les incorporent dans la Constitution. La chambre de cassation la Cour suprême a, de son coté, directement et sans devoir transiter par une disposition constitutionnelle, vérifié la conformité d'un arrêt au PIDCP ${ }^{37}$.

33 Nations Unies, Instruments internationaux relatifs aux droits de l'homme, Document de base constituant la première partie des rapports présentés par les États Parties : Burundi. HRI/CORE/1/ Add.16, 08/10/1992, para. 66 et HRI/CORE/1/Add.16/Rev. 1. (Core Document), 16/06/1992, para. 134.

34 Art. 228 de la Constitution.

35 L'article 10 de la Constitution de 1992disposait que « Les droits et les devoirs proclamés et garantis par la Déclaration universelle des droits de l'homme, les pactes internationaux relatifs aux droits de l'homme, la Charte africaine des droits de l'homme et des peuples et la Charte de l'unité nationale font partie intégrante de la présente Constitution. », voy. Décret-Loi n 1/06 du 13 mars 1992 portant promulgation de la Constitution de la République du Burundi, B.O.B., $\mathrm{N}^{\circ} 4 / 92,1$ avril 1992, p. 95 (Ici la Constitution de 1992). Il est presque l'équivalent de l'article 19 de l'actuelle Constitution. Pour une analyse de l'implication de cet article en ce qui concerne le rang hiérarchique des normes internationales des droits de l'homme en droit interne, voy. infra. pp.10-14.

36 Cour Constitutionnelle, $R C C B$ 8, arrêt du 31 mars 1993, para 8; également Cour Constitutionnelle, $R C C B$ 18, arrêt du 8 novembre 1993, Cour Constitutionnelle, $R C C B$ 54, arrêt du 5 octobre 1995 (B.O.B., $N^{\circ}$ 12/95, 1 décembre 1995, p. 427).

37 Cour Suprême, chambre de cassation, Maniraho Ange c. Ministère public, RPC 1301, Arrêt du 28 juin 2001, « que le Pacte International Relatif aux Droits Civils et Politiques qui dispose qu'on ne peut pas être témoin dans son propre procès pouvait s'appliquer dans le cas de Odette Bunama (coauteur) », publié dans Avocats Sans Frontières, Burundi. Recueil de Jurisprudence. Matières pénales, T.I, 2002, Bujumbura, p.56. 
La démarche de la Cour constitutionnelle semble suggérer un raisonnement par étapes : trouver d'abord une règle constitutionnelle et à défaut de celle-ci, -du fait peut être que le texte consacrant la norme ne fait pas partie des instruments énumérés à l'article 10 (actuellement article 19) dans le cas d'espèce-, invoquer alors l'applicabilité directe des normes internationales en droit burundais. S'il est vrai que la norme en question restera applicable par effet de l'article 19 ou non, la démarche peut avoir toute son importance. En effet, si la norme s'applique en vertu des effets du système moniste alors que l'instrument qui l'énonce n'est peut ce retrouver dans le cadre de l'article 19, son rang hiérarchique et par voie de conséquence sa portée risquent d'être affectées ${ }^{38}$.

\section{Rang hiérarchique}

Il convient à présent d'essayer de déterminer la place de la norme internationale des droits de l'homme au sein de la hiérarchie des règles juridiques en droit burundais. Cet exercice est intéressant dans la mesure où comme nous allons le voir les normes des droit de l'homme jouiraient d'un statut privilégié par rapport aux autres normes de sources internationales en ce sens que le premières seraient au même rang que les normes constitutionnelles alors que le secondes n'auraient qu'une valeur infra-constitutionnelle.

Alors que la Constitution tranche la question de l'applicabilité directe de la norme internationale, celle du rang des normes en droit positif burundais et notamment par rapport à la Constitution reste sans réponse claire. Tout au plus, la Constitution semble-t-elle indiquer des pistes de d'interprétation.

L'article 296 stipule, en effet, que,

Lorsque la Cour Constitutionnelle [...] a déclaré qu'un engagement international comporte une clause contraire à la Constitution, l'autorisation de ratifier cet engagement ne peut intervenir qu'après amendement ou révision de la Constitution.

Avant même de tenter une interprétation de la disposition aux fins de répondre à la question qui nous est posée, il faut tout de suite concéder que ce texte ne fournit qu'une indication, par ailleurs minime, puisque le texte ne fait que comparer la Constitution à un traité en cours de ratification et donc qui n'a pas encore fait son entrée en droit positif interne. A vrai dire, la question du rang entre ledit traité et la Constitution ne se pose pas tellement à ce stade. En revanche, la question est envisageable à partir du moment où ledit traité aura pris effet en droit interne et éventuellement invoqué devant le juge national.

A ce sujet et en l'absence d'un avis d'autorité donné par une haute juridiction - la Cour Constitutionnel ou la Cour Suprême-, l'on ne peut se fonder que sur certaines positions officielles ainsi que sur l'historique d'adoptions sous analyse qui, apparemment ont été di-

38 Il faut admettre que l'hypothèse d'un instrument international des droits de l'homme non applicable sous l'effet de l'article 19 paraît hypothétique puisque l'énumération des instruments que donne le texte est plutôt exemplative et non exhaustive en raison de l'usage de l'adverbe "notamment". 
rectement tirées de l'actuelle Constitution française. D'emblée, l'article 296 peut se prêter à deux lignes d'interprétation. La première soutiendrait la thèse supra-constitutionnelle des traités internationaux tandis que la seconde avancerait plutôt l'idée d'un statut infra-constitutionnel des normes inscrites dans les textes internationaux en vigueur au Burundi. Nous souscrivons à la deuxième thèse. Exception serait faite, à cet égard, des droits fondamentaux garantis par les instruments des droits de l'homme en vigueur au Burundi qui, eux, auraient le statut privilégié de normes constitutionnelles.

En première analyse, l'on peut soutenir qu'en envisageant la révision ou la modification de la Constitution afin de permettre la ratification d'un traité international, le droit positif burundais accorde, de façon implicite, une place prépondérante à la norme internationale par rapport à celle de source constitutionnelle puisque, dans le cas contraire, la Constitution aurait simplement exigé la ratification de $1^{1}$ engagement international réserve faite de la clause contraire à la Constitution ${ }^{39}$. Comme nous l'avons déjà indiqué, cependant, une analyse exclusivement fondée sur l'article 296 est d'une portée très limitée encore que le texte est, d'ailleurs, susceptible d'une interprétation dans l'autre sens comme nous allons le voir.

Il faut donc tenter de trouver des sources supplémentaires. A ce propos, certaines positions officielles semblent indicatives même si elles n'ont pas été exprimées à l'occasion d'une procédure judiciaire. Ces opinions sont, du reste, corroborées par une interprétation historique des textes concernés. S'exprimant à propos du statut du PIDCP en droit burundais lors de l'examen du rapport initial soumis par le Burundi, la délégation gouvernementale a déclaré que,

Le Pacte et la Constitution priment naturellement sur le Code Pénal et le Code de Procédure Pénale. Si ces derniers n'étaient pas en conformité avec le Pacte ou la Constitution, ils étaient automatiquement amendés; de même, toute décision en violation de la Constitution ou des droits fondamentaux était susceptible d'annulation par la Division d'Appel ${ }^{40}$.

Cette opinion compare un traité international et la Constitution d'un côté à des textes ayant valeur de lois de l'autre côté. Elle nous donne une information claire sur le rang hiérarchique des règles de sources internationale par rapport aux lois. Les premières supplantent

39 Dans ce sens, voy. Ntahiraja, B. et Nkurunziza, N. (2010) 'L'Etat face aux droits sociaux de ses citoyens: cas du droit à la santé au Burundi.', Rule of Law Program For Sub-SaharanAfrica, Librairie Africaine d'Etudes Juridiques, Vol. 7 : p.20-45.

40 Official Records of the Human Rights Committee 1992/93, Volume I, Summary records of the meetings of the forty-six session, New York, Geneva, 1996, para. 27. Texte original en Anglais, cité dans Vandenginste, S. (2011), supra, n. 94, p. 286 "The Covenant and the Constitution naturally prevailed over the Penal Code and the Code of Penal Procedure. If the latter failed to conform to the Covenant or the Constitution, they were automatically amended; furthermore, any decision which was in violation of the Constitution or fundamental humanrights would be annulled by the Appeal Division". 
les secondes ${ }^{41}$. En revanche, le commentaire ne tranche pas clairement la question du rang des normes internationales par rapport à celles ayant une valeur constitutionnelle. Quelle solution s'imposerait au juge burundais en cas de contradiction entre une norme internationale et une norme constitutionnelle?

A notre avis, la position française sur la question pourrait pertinemment inspirer le juge burundais. En effet, l'article 296 qui a été inscrit pour la première fois en droit positif burundais par la Constitution de 1992 n'est qu'une reprise de l'article 54 de la Constitution française rédigé dans des termes similaires ${ }^{42}$. Seulement, omission a été faite de l'article 55 de la Loi Fondamentale française qui précise que,

Les traités ou accords régulièrement ratifiés ou approuvés ont, dès leur publication, une autorité supérieure à celle des lois, sous réserve, pour chaque accord ou traité, de son application par l'autre partie $e^{43}$.

En droit français donc, les traités internationaux ont primauté sur les lois et règlements. En revanche, la position officielle confirmée par la jurisprudence française est que les normes internationales ont un rang inférieur à celui de la Constitution. En droit interne français donc, les normes dites du «bloc constitutionnel ${ }^{44}$ prime sur toute autre y compris celles ayant pour source un traité international. Le droit positif français place la norme internationale hiérarchiquement entre la Constitution et la $\operatorname{loi}^{45}$.

41 Le Burundi a réitéré cette position dans le cadre du rapport initial soumis à la Commission Africaine des Droits de l'Homme et des Peuples en vertu de la Charte Africaine « L'Etat du Burundi reconnait que les normes internationales auxquelles il a souscrit sont hiérarchiquement supérieures à ses lois et règlements. ». Voy. Commission Africaine des Droits de l'Homme et des Peuples, Rapport initial du Burundi, DOC/OS(XXVII), 154c, 4 mars 2005, para. 15.

42 L'art. 296 précité est une transposition de 1'art. 54 de la Constitution française « Si le Conseil Constitutionnel, saisi par le Président de la République, par le Premier Ministre, par le Président de l'une ou l'autre assemblée ou par soixante députés ou soixante sénateurs, a déclaré qu'un engagement international comporte une clause contraire à la Constitution, l'autorisation de ratifier ou d'approuver l'engagement international en cause ne peut intervenir qu'après révision de la Constitution. », accessible en le site web du Gouvernement français, le service public de la diffusion du droit en suivant le lien : http://www.legifrance.gouv.fr/Droit-francais/Constitution/Constitution-du -4-octobre-1958\#ancre2178_0_7_, consulté le 14/07/2013.

43 Art. 55 de la Constitution française.

44 Le bloc constitutionnel comprend :

1) le préambule et les articles de la Constitution du 4 octobre 1958;

2) le préambule de la Constitution de 1946;

3) la Déclaration des droits de l'homme et du citoyen de 1789;

4) la charte de l'environnement;

5) les principes fondamentaux reconnus par les lois de la République;

6) les principes et objectifs de valeur constitutionnelle.

45 Conseil d'État (Ass., 20 octobre 1989, Nicolo, n 108243); Cour de cassation (Ch. mix., 24 mai 1975, Société des cafés J. Vabre, $n^{\circ}$ 73-13556). Commentés dans ibid. « Le Conseil d'État et la Cour de cassation, par application de l'article 55 de la Constitution, veillent à la prééminence du droit international, y compris le droit dérivé, sur les lois et règlements » (...) «Issues des engage- 
L'on pourrait d'ailleurs aboutir à la même conclusion abstraction faite de l'article 55 de la Constitution française qui n'a pas été reprise dans notre droit. Par la seule interprétation de l'article 54 presqu'équivalent de notre article 296, l'on peut, en effet, avancer que les normes internationales sont subordonnées à la Constitution puisqu'elles ne peuvent produire d'effet juridique si elles lui sont contraires ${ }^{46}$.

Nous pensons que le juge burundais pourrait s'inspirer utilement de ces dispositions et opinions pour donner primauté aux normes internationales régulièrement introduites dans le droit burundais sur les lois et règlements à l'exclusion des règles à valeur constitutionnelle qui, elles, lui sont supérieures. A cet égard, les normes des droits de l'homme jouiraient ainsi d'un statut privilégié par rapport à celles contenues dans d'autres textes internationaux en vigueur puisqu'elles auraient une valeur constitutionnelle en droit burundais par effet de l'article 19. C'est ce que suggère, encore une fois, une interprétation analogique des dispositions pertinentes des droits français et burundais. La Constitution française, énumère, en effet, les règles qui font partie $\mathrm{du}$ « bloc constitutionnel ». Ces règles ont valeur constitutionnelle et sont donc supérieures à toute autre règle. En tant que telle, la Déclaration des Droits de l'Homme et du Citoyens, est citée parmi celles-ci ${ }^{47}$. Par analogie, l'on peut suggérer que, les normes garanties par les instruments des droits de l'homme en vigueur au Burundi devraient bénéficier du même traitement en vertu de l'article 19 de la Constitution qui dispose que,

Les droits et devoirs proclamés et garantis, entre autres, par la Déclaration universelle des droits de l'homme, les Pactes internationaux relatifs aux droits de l'homme, la Charte africaine des droits de l'homme et des peuples, la Convention sur l'élimination de toutes les formes de discrimination à l'égard des femmes et la Convention relative aux droits de l'enfant font partie intégrante de la Constitution de la République du Burundi.

Ce texte conférerait ainsi une valeur constitutionnelle aux normes des droits de l'homme en droit burundais.

\section{Le mouvement des droits de l'homme au Burundi}

Cette section traite du mouvement de revendication des droits de l'homme au Burundi. Une attention particulière sera réservée à trois lacunes dans les programmes et stratégies d'action des ONGs de défense des droits de l'homme qui justifieront les recommandations de

ments internationaux de la France et régulièrement introduites dans notre droit, elles s'imposent à toutes les normes de droit interne excepté celles qui ont valeur constitutionnelle ».

46 Dans ce sens, Gouvernement français, Direction de l'information légale et administrative, Découverte des Institutions, Approfondissements, Les Traités Internationaux et la Constitution, accessible en suivant le lien : http://www.vie-publique.fr/decouverte-institutions/institutions/approfondi ssements/traites-internationaux-constitution.html, consulté le 14/07/2013.

47 Voy. supra, n.43. 
l'article. La première consiste, comme l'avons mentionné dans l'introduction, dans l'absence d'une culture de revendication judiciaire des droits. La deuxième est liée à des programmes et stratégies d'intervention peu adaptés aux défis locaux. Pour être plus précis à ce sujet, un focus presqu'exclusif sur les droits civils et politiques tel qu'il est actuellement le cas au détriment des droits économiques, sociaux et culturels ne peut pas assurer l'émancipation des masses afin qu'elles s'approprient la revendication des droits de l'homme.

\section{Absence d'une culture de revendication judiciaire des droits}

La première limite communément identifiée par les défenseurs des droits de l'homme euxmêmes dans leurs interventions consiste en l'absence d'une culture de revendication des droits par les citoyens. Ce défi serait dû, selon eux, à l'histoire très récente de la société civile au Burundi. En effet, le mouvement de défense des droits de l'homme au Burundi ne remonte qu'aux années 1990 avec le processus de démocratisation des institutions et de libéralisation de l'espace politique. Les premières organisations de défense des droits de l'homme virent le jour avec l'adoption, en $1992^{48}$, de la première Constitution autorisant le multipartisme et établissant les principes d'un système démocratique pour la première fois dans l'histoire post- coloniale du Burundi.

C'est un fait avéré que malgré son affirmation, le principe de l'applicabilité directe de la norme internationale que nous avons eu l'occasion d'explorer n'est pas traduit en une réalité dans la pratique judiciaire burundaise. Il est, en effet, rare que les justiciables invoquent les sources internationales des droits de l'homme ou que le juge s'en inspire. Gilles CISTAC, par exemple, dans son étude sur la pratique en matière de justice administrative, relève que par "principe de proximité », les praticiens recourent, parfois sans discernement, aux règles de droit interne les plus immédiates au détriment ou au mépris d'une ouverture utile aux sources internationales ${ }^{49}$. Analysant la jurisprudence de la Cour Constitutionnelle, Vandenginste, de sa part, arrive à la conclusion selon laquelle rarement la Cour n'a que rarement servi de cour des droits de l'homme malgré le fort potentiel qu'elle pourrait présenter dans la protection des droits de l'homme en application des standards internationaux ${ }^{50}$.Il fait, en effet, remarquer que la Cour n'a été que rarement saisie de requêtes par des particuliers, individus ou personnes morales, invoquant des violations des droits de l'homme excepté pour la période couvrant ses premières années d'existence ${ }^{51}$.

48 Voy. supra, n. 10.

49 Cistac, G. (2008), supra, n. 10, p.121.

50 Voy. Vandenginste (2011),supra, n. 10, Chap. 6, applying international law and reducing political expediency through constitutional adjudication, p. 359.

51 Vandenginste analyse la jurisprudence de la Cour sous l'empire de la Constitution du 18 mars 1992, supra., n. 102; de l'Acte Constitutionnel de Transition de juin 1998, Décret-Loi n 1/008 du 6 juin 1998 portant promulgation de l'Acte Constitutionnel de Transition de la République du Burundi, B.O.B., $n^{\circ} 7 / 98,1$ juin 1998, p.468; de la Constitution de Transition du 28 octobre 2001, Loi $\mathrm{n}^{\circ} 1 / 017$ du 28 octobre 2001 portant promulgation de la Constitution de la République du Burundi, 
Le peu d'attention que les individus et les ONGs réservent au mécanisme constitutionnel de protection des droits de l'homme peut s'expliquer par plusieurs facteurs. Premièrement, une évolution en matière de procédure et de jurisprudence de la Cour a abouti à des restrictions quant à la saisine de la Cour par les particuliers. Une des restrictions importantes porte sur les textes susceptibles d'être attaqués sous le régime de l'actuelle Constitution. Contrairement aux textes constitutionnels antérieurs, la Constitution du 18 mars 2005 a été interprétée dans le sens d'interdire aux particuliers (personnes physiques ou personnes morales) d'attaquer les actes réglementaires autres que ceux pris en exécution de la loi. Cette restriction a eu des conséquences pratiques conduisant à des arrêts systématiques

B.O.B., $n^{\circ}$ 10/2001, 1 octobre 2001, p. 1269 ainsi que de la Constitution du 18 mars 2005. Entre 1992 et 1996 (abolition de la Cour Constitutionnelle), la Cour avait déjà rendu 56 arrêts dont la moitié portait sur des requêtes émanant des individus ou personnes morales. Un bon nombre de ces arrêts faisaient, par ailleurs, explicitement référence à des droits énoncés par des instruments des droits de l'homme. La Cour a, par exemple, déclaré un Décret-Loi contraire à l'art. 26 de la Constitution (liberté d'opinion et de presse), Cour Constitutionnelle, $R C C B$ 6, Arrêt, 17 août 1992; à deux autres occasions, elle (la Cour) a estimé que certaines dispositions du Décret-Loi portant création de la Cour des Comptes étaient contraires à l'art. 10 de la DUDH et ainsi qu'à l'art. 14, para. 1 du PIDCP (droit à un procès équitable), Cour Constitutionnelle, $R C C B$ 8, Arrêt, 31 mars 1993 et Cour Constitutionnelle, $R C C B$ 18, Arrêt, 8 novembre 1993 (supra, n. 102). Dans les deux cas, la Cour a également vérifié si le texte attaqué était en violation ou non de l'art. 11 de la DUDH (présomption d'innocence); par l'arrêt $R C C B 47$ du 18 novembre 1994, la Cour a déclaré que le Décret-Loi portant nationalisation de la compagnie Burundi Tobacco company était en violation de l'art. 27 de la Constitution (droit à la propriété); de même, elle a estimé qu'une loi d'amnistie adoptée en septembre 1993 était en violation du principe de non discrimination énoncée par l'art. 15 de la Constitution, Cour Constitutionnelle, RCCB 54, Arrêt, 5 octobre 1995. Les arrêts de la Cour étaient, dans la plupart des cas, basés sur des articles de la Constitution même si les requérants invoquaient également des instruments internationaux. La Cour estimait que dès lors qu'elle avait pu trouver une violation du droit invoqué en se fondant sur une base constitutionnelle, il n'était plus nécessaire de vérifier une éventuelle violation du même droit tel que garanti par tel ou tel autre instrument international, confirmant ainsi implicitement l'applicabililité du droit international des droits de l'homme. Dans ce sens et pour plus de détails, voy. Vandenginste (2011),p. 385. Par la suite, c'est-à-dire sous l'empire des textes constitutionnels postérieurs à la Constitution de 1992, les requêtes individuelles de même que les arrêts faisant référence aux droits de l'homme ne sont restés que très exceptionnels. Pour plus de détails, voy. Vandenginste (2011), pp. 389-394. Même à considérer cette période pendant laquelle Vandenginste estime que la Cour Constitutionnelle a pu fonctionner comme une cour des droits de l'homme, la portée en reste limitée par rapport à l'objet de notre étude compte tenu notamment du profil des requérants. Les requérants n'étaient généralement des ONGs. Ils étaient souvent des personnalités politiques. Ce qui a, d'ailleurs, amené une certaine opinion à considérer la Cour comme un instrument de l'opposition politique. Reyntjens, par exemple, parle d'une " véritable guérilla juridique », qui opposait le Frodebu (parti au pouvoir à l'époque à l'issue des élections de 1993) et la Cour Constitutionnelle. Voy. Reyntjens, F. 'Justice et politique : la Cour Constitutionnelle' (1995) in Guichaoua, A. (ed.), Les crises politiques au Rwanda et au Burundi. Paris : Karthala, pp. 173-175; Reyntjens, F. (1996) Burundi : Briser le cycle de violence. Londres : Minority Rights Group International, p. 17. Il est au-delà de l'objet de notre travail d'entrer dans ces débats. 
d'inadmissibilité de telles requêtes ${ }^{52}$. Or, ce sont justement les actes administratifs qui sont souvent susceptibles de violer les droits des particuliers au quotidien. Deuxièmement et corollairement, il existe une opinion au sein du public selon laquelle, dans son contexte actuel de fonctionnement, la Cour n'est qu'un instrument de l'exécutif (parfois du législatif même $)^{53}$.Il s'en est suivi un désintéressement et une sorte de résignation, les justiciables étant convaincus d'avance que toute procédure devant la Cour échouerait inévitablement.

Vandenginste estime que,

Cela pourrait être remédié par une campagne d'information en l'endroit des membres du parlement, des organisations de la société civile, des avocats et des étudiants en droit, qui tous devraient être mieux informés du rôle crucial de la Cour Constitutionnelle. Ils devraient également éliminer ce que l'on pourrait appeler une "culture de résignation " et refuser d'accepter, malgré la preuve malheureuse a contrario, que la Cour Constitutionnelle se trouve simplement au service de ceux qui sont au pouvoir ${ }^{54}$.

Nous sommes tout à fait d'accord avec Vandenginste au sujet de ces recommandations. Nous pensons, néanmoins, que dans le contexte burundais, simplement informer les acteurs et les citoyens de leurs droits et des cadres légaux et institutionnels (mêmes efficaces) pour les défendre ne suffirait pas à éradiquer cette « culture de résignation » pour développer une culture de revendication. Il s'agit là, en effet, d'une approche presque en droite ligne avec

52 Voy. Par ex. Cour Constitutionnelle, $R C C B$ 209, Arrêt, 31 décembre 2007 « Attendu qu'il ressort de ce qui précède que le législateur, dans le souci de conformer la loi à la Constitution, a entendu limiter le droit de saisine de la Cour Constitutionnelle pour les personnes physiques notamment aux seules lois entendues stricto sensu à l'exclusion des actes réglementaires tels que les décrets, ordonnances, etc. ». En l'espèce, un groupe d'étudiants de l'Université du Burundi attaquaient une ordonnance ministérielle. La Cour confirmait ainsi sa position dans l'affaire $R C C B 174$ dans laquelle un groupe d'ONGs mettaient en cause une ordonnance ministérielle portant libération des prisonniers dits politiques. L'espace ne nous permet pas de discuter de cette ligne d'interprétation mais il s'agit, à notre avis d'une interprétation trop restrictive et erronée dans le cadre d'un contentieux sur les droits de l'homme.

53 Pour l'exemple d'un arrêt commenté dans ce sens, voy., Vandenginste, S. (2008) ' Pouvoir et droit au Burundi : un commentaire (principalement) juridique spécifiquement une analyse sur l'arrêt du 5 juin 2008 de la Cour Constitutionnelle dans l'affaire RCCB 213', Dialogue, № 243, pp. 45-65.

54 "This maybe remedied by an awareness campaign among members of parliament, civil society groups, practicising attorneys and law students, all of whomshouldbebetterinformed of about the crucial role of the Constitutional Court. They should also shed what maybe called a 'culture of resignation' and refuse to accept, despite unfortunate evidence a contrario, that the Constitutional Court merely rules at the service of those holding power.", VANDENGINSTE (2011), p. 413. Nous adhérons particulièrement à l'idée qu'il ne faudrait pas que les justiciables abandonnent l'exercice des recours judiciaires au motif que les institutions qui les mettent en œuvre fonctionnent mal. Intensifier les saisines constituerait plutôt un des moyens pour les bousculer et éventuellement les amener à une évolution jurisprudentielle. Sur l'importance d'un tel exercice en matière de protection constitutionnelle des droits de l'homme dans les contextes africains, voy. ABDULLAHI, A. (2003), supra., n.119, p.16. 
des actions autour des droits civils et politiques. Or, nous sommes d'avis qu'elle d'effets limités dans le contexte burundais. Il faut, en plus de cela, une adaptation des actions et stratégies actuelles d'intervention en matière de défense des droits de l'homme.

Actions et stratégies d'intervention peu adaptées aux défis locaux

Si on devait énumérer les principales lignes d'action des acteurs burundais dans le domaine des droits de l'homme, l'on identifierait les suivantes : enquêter et dénoncer les violations des droits et libertés fondamentales, mener des activités de plaidoyer et, dans une certaine mesure informer les individus de leurs droits et fournir des services d'assistance juridique. Il faut tout de suite faire remarquer que surveiller la situation des droits de l'homme pour ainsi identifier et dénoncer les cas de violation constitue l'activité principale des ONGs.

L'on comprend l'importance accordée à ce type d'actions si on resitue le mouvement burundais de défense de droits de l'homme dans ses origines. Tout comme le mouvement africain général, il émane directement du mouvement européen répandu à travers le monde avec le triomphe de la démocratie libérale d'origine occidentale ${ }^{55}$. Le processus de diffusion des standards internationaux des droits de l'homme est intimement lié à la campagne de démocratisation entreprise avec la fin de la Guerre Froide ${ }^{56}$.C'est dans ce sens qu'il faut comprendre l'universalisation des constitutions libérales des années 1990, le Burundi se retrouvant dans ce courant général.

Les ONGs burundaises de défense des droits de l'homme qui ont vu le jour dans les 1990sont alors simplement adopté les stratégies classiques d'intervention de leurs homologues du Nord ${ }^{57}$. En plus de ces activités classiques et communes aux ONGs de défense

55 Pour une réflexion sur la dimension idéologique des droits de l'homme, voy. MUTUA, M., (1995-1996) The ideology of humanrights, 36 Va. J. Int'l L.: 589-659. Mutua (id., p. 592) estime que le discours sur les droits de l'homme constitue un des moyens de propagation des valeurs et principes de la démocratie libérale d'origine occidentale à travers le monde.

56 Alford, William, P. (2000) "Exporting "the pursuit of happiness", Harvard Law Review, 113: 1677-1715. D'après Mutua, promotion des droits de l'homme et celle de la démocratie politique étaient employées de façon interchangeable. Voy. Mutua, M. (Apr. 2008) "Human rights in Africa: the Limited Promise of Liberalism", AfricanStudiesReview, vol. 51, $\mathrm{N}^{\circ} 1:$ p. 22.

57 La technique d'enquête sur les violations des droits de l'homme telle que pratiquée par des ONGs des droits de l'homme consiste à effecteur des missions d'enquête, collecter des informations à confronter avec les standards internationaux, identifier les écarts par rapport à ces standards, rédiger des rapports à disséminer. Cette dernière méthode est également appelée technique du «blâme » (shaming) puisqu'elle vise à identifier les Etats prédateurs et à susciter une désapprobation de leur comportement sur la scène internationale. Sur la technique d'enquête, Voy. Orentlicher, Diane F. (1990) "Bearing Witness: The Art and Science of Human Rights Fact-Finding", Harvard Human Rights Journal, vol. 3, pp. 83-135, également cité dans Mutua, M., (1995-1996), supra, n.120, p. 621; sur celle du rapportage, voy., par ex.Mutua, M., (1995-1996), supra, n.120. Mutua fait allusion aux activités des ONGs comme le Comité des Juristes pour les Droits de l'Homme. 
des droits de l'homme, certaines élaborent des programmes de promotion de l'état de droit en général ${ }^{58}$.

Ces politiques d'intervention trouvent leur fondement dans la tradition libérale classique. La thèse libérale des droits de l'homme repose sur le postulat selon lequel les citoyens (qui remplissent les conditions) doivent avoir l'occasion de participer dans la direction des affaires de leur pays notamment à travers l'organisation périodique d'élections libres et crédibles ${ }^{59}$. De même, un système démocratique implique la reconnaissance d'un certain nombre de libertés fondamentales essentiellement en termes droits civils et politiques : liberté d'expression, de rassemblement, d'association, etc. ${ }^{60}$

En vertu de cette conception de la démocratie et des obligations qui en découlent pour l'Etat, celui-ci est tenu de mettre en place des mécanismes légaux et institutionnels nécessaires à l'exercice des droits et libertés fondamentaux par les individus. L'existence de ces mécanismes suffirait pour que les bénéficiaires des droits les exploitent.

Une des limites de ces conceptions traditionnelles de la démocratie libérale, commente le professeur Mutua, est qu'elles n'imposent pas à l'Etat l'obligation de corriger d'éventuelles inégalités qui pourraient prévaloir au sein de la société et limiterait ainsi le potentiel de l'exercice des droits ainsi garantis pour certains ${ }^{61}$. L'Etat, poursuit- il, met en place des mécanismes dont il ne revient qu'aux citoyens d'explorer. Si les opportunités et l'effectivité de la participation peuvent être limitées pour certains en raison du manque de ressources financières ou de leur statut social,

[...] il n'est pas de la responsabilité de l'Etat d'atténuer cette dépendance, d'ouvrir des voies à la participation politique qu'un manque de ressources économiques ou d'éducation ou de statut pourrait autrement entraver ${ }^{62}$.

La justice sociale n'est pas de l'essence de cette approche libérale. C'est dans ce sens qu'il faut comprendre la place privilégiée réservée aux droits civils et politiques au détriment des droits économiques, sociaux et culturels dans la tradition occidentale des droits de l'homme et partant dans le processus d'élaboration des standards internationaux ${ }^{63}$.

58 Pour plus de commentaire, voy. Mutua, id., p. 621.

59 Huntington, Samuel P. (1991) The Thirdwave: Democratization in the Twentieth Century. Norman: University of Oklahoma City. Commenté dans Mutua, M. (2008), ibid., pp. 20-21.

60 Huntington, Samuel P. (1991), Ibid.

61 Mutua (2008), p. 21.

62 “...it is not the government's responsibility to alleviate that dependence, to open paths to political participation which lack of funds or education or status would otherwise block". Steiner, Henry P. (1998) "Political Participation as a Human Right.", Harvard Human Rights Yearbook, pp. 109-110; également cite dans Mutua (2008), pp. 21-22.

63 Par exemple, aucun organe chargé de surveiller l'application du PIDESC contrairement au PIDCP dont l'adoption état suivi de la création du Comité des droits de l'homme en tant qu'organe de surveillance. Selon Mutua, le premier était par ailleurs délibérément rédigé dans un langage vague qui devait handicaper sa mise en application. Voy. Mutua, M. (2007) Standard Setting in Human Rights: Critique and Prognosis, Human Rights Quarterly, Vol. 29, 3, pp. 547-630. Pour une ana- 
Compte tenu de leurs origines et de leurs racines dans les Etats de tradition libérale, les Organisations Internationales Non Gouvernementales (OINGs) les plus réputées dans le mouvement mondial des droits de l'homme se sont, elles aussi, peu préoccupées des droits économiques, sociaux et culturels ${ }^{64}$. En règle générale, ces organisations ont presqu'exclusivement centré leurs interventions sur les droits civils et politiques ${ }^{65}$.

De leur part et par mimétisme, les ONGs burundaises les plus visibles dans le domaine des droits de l'homme n'ont pas de programmes clairement orientés vers la promotion des droits économiques, sociaux et culturels ${ }^{66}$.Cela est lié à leur dépendance morale des ONGs

lyse détaillée sur les débats idéologiques autour de l'élaboration du PIDESC et notamment sur la manière dont les Etats-Unis et les puissances occidentales étaient opposés aux droits économiques, sociaux et culturels qu'ils lient avec le droit au développement, voy. Alston, P. and Quinn, G., (May. 1987) "The Nature and Scope of States Parties' Obligations under the International Covenant on Economic, Social and Cultural Rights, Human Rights Quarterly vol. 9, N. 2: pp. 156-229. Mutua (2007), p.618 fait remarquer que des auteurs de renom et de tradition occidentale ont sévèrement critiqué les droits économiques, sociaux. Il donne pour exemple: Donnelly, J., (1985) In Search of the Unicorn: The Jurisprudence and the Politics of the Right to Development, 15 CAL. W. INT'L L. J., pp. 473-482 (1985) et Marks, S. (1981) "Emerging Human Rights: A New Generation for the 1980s?", Rutgers law review, 33, pp. 435-451. Marks écrivait que les droits de la troisième génération, dont le droit au développement reste le plus dominant sont « trop vague pour être justiciables et ne sont que des slogans. Donnelly, quant à lui, soutenait que les droits collectifs étaient « fallacieux ", " désorientés » et " dangereux ». Pour une réponse à ceux qui s'opposent à un droit au développement, voy. Alston, P. (1988) "MakingSpace for New Human Rights: The Case of the Right to Development", Harvard Human Rights Yearbook, 1, 3, pp. 29-30.

64 Ces OINGs sont soit d'origine américaine soit occidentale. Les organisations suivantes sont couramment citées comme les plus dominantes sur la scène internationale : Amnesty International (Londres), Human Rights Watch (New-York), Comité des Juristes pour les droits de l'homme (actuellement Human Rights First, New York), International Law Group (actuellement Global Rights, Washington, D.C.) ainsi que la Commission Internationale des Juristes (Genève). Voy. Henry, Steiner J. (1991) Diverse partners: Non-Governmental Organizations in the human rights movement: Harvard Law School Human Rights Program and Human Rights Internet, pp. 19-22; Mutua, M. (2009) "Standard Setting in Human Rights: Critique and Prognosis", Human Rights Quarterly, Vol. 29, 3, p.591.

65 Mutua, M., ibid.p. 592; Mutua, M. (2008) "Human Rights and Powerlessness: Pathologies of Choice and Substance", Buffalo Law Review, 56, pp. 1025-1034. Mutua relève, néanmoins, une ONG américaine des droits de l'homme qui se focalise sur les droits économiques, sociaux, culturels : The Centre for Economic and Social Rights (SERAC, de création plutôt récente, 1993). SERAC vise la promotion de la justice sociale à travers les droits de l'homme. Mutua note cependant que des OINGs comme Human Rights Watch et Amnesty International ont commencé à payer une certaine attention aux droits économiques, sociaux et culturels auxquels ils s'opposaient autrefois, Mutua, M. (2009), id., p. 592. Sur la manière dont les OINGs devraient promouvoir les droits économiques, sociaux et culturels, voy. Leonard S. Rubenstein, (Nov., 2004) "How International Human Rights Organizations Can Advance Economic, Social, and Cultural Rights: A Response to Kenneth Roth", Human Rights Quarterly, Vol. 26, No. 4, pp. 845-865.

66 Interview avec des cadres de la Ligue "ITEKA" et de l'APRODH, respectivement le 29 et le 30 juin 2013. La même situation se retrouve dans d'autres contextes africains. Pour les cas de l'Uganda, la Tanzanie et le Kenya, voy.Voy. par ex.,Loka-Onyango, J., "NGO Struggle for Economic, Social and Cultural Rights in UTAKE: A Ugandan Perspective, in Mutua (2008), id., chap. 4, p. 75. UTAKE est l'abréviation de Uganda, Tanzanie et Kenya. Les auteurs analysent le mouvement 
« partenaires » dont elles s'inspirent aveuglement ${ }^{67}$.A cette dépendance morale s'ajoute une dépendance financière. Les ONGs burundaises de défense des droits de l'homme sont fiancées par des bailleurs de fonds étrangers généralement occidentaux-tout comme la plupart des ONGs du sud. Elles doivent, pour cela, aligner leurs activités sur les politiques de financement de ces bailleurs. Le résultant étant toujours la marginalisation des droits économiques, sociaux et culturels ${ }^{68}$.

En Afrique et au Burundi en particulier, la réalisation des droits économiques, sociaux et culturels conditionne, pourtant, l'exercice effectif des droits y compris les droits civils et politiques. Des défis structurels liés notamment à la pauvreté et à l'analphabétisme, etc. minent, en effet, le potentiel des populations africaines à défendre leurs droits. A ce sujet, les observations communément faites en ce qui concerne le contexte africain en général sont, dans une large mesure, valables dans le cas du Burundi. Odinkalu, par exemple, soutient que la dépendance dont souffrent les ONGs du sud les déconnecte des réalités locales et ne leur permet pas de développer un mouvement de défense des droits de l'homme enraciné dans les communautés à la base ${ }^{69}$.

Pourtant, comme Abdullah An-Na'im le souligne pertinemment, l'ultime remède aux violations des droits de l'homme en Afrique, « dépend de la volonté et de la capacité des populations elles-mêmes de s'opposer, au degré le plus maximal, à l'oppression et aux vio-

des droits de l'homme ces pays de l'Afrique de l'Est. Encore une fois, conclusions qu'ils tirent sont valables, pour l'essentiel, dans le contexte burundais.

67 Citant Philip Alston qui critiquait le fait qu'une OING comme Amnesty International mondialement influant s'est exclusivement focalisée sur les droits civils et économiques, Mutua fait observer que la plupart des ONGs du sud s'en sont malheureusement inspirées. Alston, P. (1990) The Fortieth Anniversary of the Universal Declaration of Human Rights: A Time More for Reflection than for Celebration, in Human rights in a pluralist world:individuals and collectivities 1, 9 (Jan Berting et al. eds.). cité dans Mutua, M. (2009), id., p.593.

68 Pour une même observation concernant le Kenya, Voy. Kanyiga, K. (2008) Contradictions in Neoliberalism: Donors, Human Rights NGOs, and Governance in Kenya, chap. 8, p. 183 in MutuaMakau (ed.) Human Rights NGOs in East Africa : Political and Normative Tensions. Philadelphia: University of PennsylvaniaPress. Kanyinga fait remarquer que les ONGs du sud ont promu des valeurs néolibérales au détriment des questions socioéconomiques pourtant particulièrement pertinentes dans les contextes africains; également MAINA, P. (2008) en ce qui concerne la Tanzanie. Citant Sivji, Maina écrit: « Nous sommes financés et dépendons presqu'exclusivement sur le financement extérieur (...). Directement ou par des moyens subtiles, ceux qui nous financent déterminent, limitent ou réorientent nos agendas ", "We are funded by, and relyalmostexclusively on, foreignfunding. (...) In many direct and subtle ways, those who fund us determine or place limits on our agendas or reorient them." Maina, P. (2008) "Conclusion: Coming of Age: NGOs and State Accountability in East Africa. In MakauMutua (ed.), p. 310.

69 Odinkalu, Chidi A. (2000) Why More Africans Don't Use Human Rights Language, Human Rights Dialogue, 3-4. Cité dans Mutua, M. (2009), supra, n.129, p.594. Les activistes burundais sont conscients de leur déconnection des masses qu'ils prétendent représenter. Voy. Nkurunziza, N. (2012) The Potential for, and Limits to an Approach "From Below" to Transitional Justice in the Burundian Context. Master Dissertation, Trinity College Dublin, Dublin, p.69, Interview avec un représentant de l'ONG Réseau Femmes et Paix, Bujumbura, 23 juillet 2012. 
lations de leurs droits $»^{70}$. Il faut, pour cela, développer une culture de résistance ${ }^{71}$, une citoyenneté active comme le mentionne Odinkalu ${ }^{72}$. Cela requiert des acteurs des droits de l'homme une prise au sérieux du principe d'interdépendance des droits de l'homme avec plus d'attention aux droits économiques, sociaux et culturels qu'il n'en est actuellement le cas. Insistance devrait être faite sur des droits de base comme le droit à l'éducation. ${ }^{73}$. Ces droits sont, précise Shedrack, pour les Africains, les "premiers moyens d'auto-défense", leur seule voie pour surmonter les défis structurels et ainsi prendre à leur compte le combat pour la dignité humaine qui se trouve au centre des idéaux des droits de l'homme ${ }^{74}$.

\section{CONCLUSION}

La présente avait pour objectif d'analyser la contribution des ONGs dans le mouvement de défense des droits de l'homme au Burundi en se focalisant sur la revendication judiciaire des droits de l'homme. Partant du constat de l'absence d'une culture dans ce sens dans le contexte burundais, l'argument principal de l'article a essentiellement consisté en une recommandation d'adaptation des programmes et stratégies d'action des ONGs afin d'intégrer cette approche dans leurs lignes d'intervention. A cet effet, il a été recommandé que ces organisations accordent plus d'attention à la promotion des droits économiques, sociaux et culturels. Ceux-ci se trouvent actuellement défavorisés par rapport aux droits civils et politiques. En effet, quoique ces derniers méritent également une attention, une solution durable aux violations des droits de l'homme ne peut venir que d'une émancipation des masses afin que les populations soient équipées pour prendre en main le combat pour leur dignité. Or, la promotion de certains droits de base de la catégorie des droits économiques, sociaux et culturels en constitue le tremplin.

70 Abdullahi, A. (2003), supra. n. 119, p. 3.

71 La notion de psychologie et sociologie de la résistance que développe cet auteur repose sur l'idée selon laquelle les Etats, auteurs des violations des droits de l'homme sont des calculateurs. Ils tiennent compte d'une éventuelle résistance des victimes de violations des droits avant d'entreprendre toute action (portant atteinte aux droits de l'homme),Abdullahi, A. (2003), supra., n. 119., p.8.

72 Odinkalu, C. Anselm (2003) Back to the Future: The Imperative of Prioritizing for the Protection of Human Rights in Africa, Journal of African Law, Vol. 47, No. 1, pp. 1-37.

73 Voy. dans ce sens Odinkalu (2003), supra, n. 137, p. 4.

74 Shedrack, c. Agbakwa $†$ (2002) "Reclaiming Humanity: Economic, Social, and Cultural Rights as the Cornerstone of African Human Rights", Yale Human Rights \& Development L. J., Vol. 5. Mutua estime que les ONGs des droits de l'homme du sud ne pourront y parvenir que si elles adoptent des stratégies innovantes afin de s'affranchir de leur dépendance de l'Occident. Elles devraient chercher à galvaniser des soutiens financiers et moraux locaux, Makau Mutua, M (ed.) (2008), p. 5; dans le même sens, Sewanyana estime que seule une société civile puissante de l'intérieur peut être un agent de changement social important. L'auteur pense que sans toutefois renoncer à leur indépendance, les organisations de la société civile pourraient envisager des relations de partenariat avec les gouvernements locaux; Sewanyana, L. (2008), in Makau Mutua (ed.), chap. 9 "State and Civil Society Relations: Constructing Human Rights Groups for Social Change”. 
Avant de passer à l'argument de l'article, une section préliminaire a été réservée à l'analyse du cadre juridique et institutionnel de protection des droits de l'homme au Burundi. Cet exercice nous a permis d'identifier les obligations qui s'imposent à l'Etat du Burundi en vertu de ses engagements internationaux en matière des droits de l'homme ainsi que les mécanismes mis en place pour remédier à d'éventuelles violations. Il a été démontré que, sur le plan théorique, le Burundi offre un cadre normatif et institutionnel relativement propice à la revendication judiciaire des droits. En effet, les normes internationales sont directement invocables devant le juge national. Celles des droits jouissent même d'un statut privilégié puisqu'elles sont de rang constitutionnel. La Cour Constitutionnelle est la plus haute juridiction compétente pour garantir le respect de la Charte des droits et libertés fondamentaux par les autorités administratives et judiciaires.

\section{BIBLIOGRAPHIE}

I. Textes internationaux et Régionaux

1. Pacte International Relatif aux Droits Civils et Politiques. In Nations Unies, Recueil des Traités, vol. 999, p. 171 et vol. 1057, p. 407.

2. Pacte International Relatif aux Droits Economiques, Sociaux et Culturels. In Nations Unies, Recueil des Traités, vol. 993, p. 3.

3. Charte Africaine des Droits de l'Homme et des Peuples, O.A.U. Doc. CAB/LEG/67/3 rev. 5. In21 I.L.M. 58 (1982).

II. Textes législatifs et règlementaires internes

1. Loi $\mathrm{N}^{\circ} 1 / 010$ du 18 mars 2005 portant promulgation de la Constitution de la République du Burundi, B.O.B 2005, $n^{\circ} 3$ Ter, pp. 1-35.

2. Loi $\mathrm{n}^{\circ} 1 / 017$ du 28 octobre 2001 portant promulgation de la Constitution de la République du Burundi, B.O.B., $n^{\circ}$ 10/2001, 1 octobre 2001, p. 1269.

3. Décret-loi $\mathrm{n}^{\circ} 1 / 008$ du 6 juin 1998 portant promulgation de l'Acte Constitutionnel de Transition de la République du Burundi, B.O.B., $n^{\circ} 7 / 98,1$ juin 1998, p.468.

4. Décret-loi $\mathrm{n}^{\circ} 1 / 06$ du 13 mars 1992 portant promulgation de la Constitution de la République du Burundi, B.O.B., $N^{\circ}$ 4/92, 1 avril 1992, p.95.

III. Ouvrages et contribuions dans des ouvrages

1. ABDUllaHI, A. (2003) Expanding Legal Protection of Human Rights in African Contexts.In AN-NA'IM, ABDULLAHI (ed.), Human Rights Under African Constitutions, Realizing the Promise for Ourselves, Philadelphia: University of Pennsylvania Press, p. 3. 
2. ANKUMAH, E. (2008) 'The African Commission on Human and Peoples' Rights'. In EVANS and MURRAY (eds.), The African Charter on Human and People's Rights: The System in practice, $2^{\text {nd }}$ ed. Cambridge: Cambridge University Press, p.93.

3. BARICAKO, G. (2008) The African Charter and African Commission on Human and Peoples' Rights. In Evans, Malcolm and Murray, Rachel (eds.), pp. 1-19.

4. BOVAY, N. and PROUVEZ, N. (2001) The role of the International Commission of Jurists in promoting human rights under the rule of law. In C. E. WELCH (ed.), $N G O s$ and Human Rights, pp. 119-140.

5. DONNELLY, J. (2007) Universal Human Rights in Theory and Practice. Oxford: Westview Press.

6. DRZEWICKI, K. (1999) 'Internationalization of Human Rights and their Juridization'. In RAIJA HANSKI and MARKKU SUKSI (eds.), Revised Edition, An Introduction to the International Protection of Human Rights: A Textbook, p. 31.

7. EVANS, M. and MURRAY, R. (2008) The State Reporting Mechanism of the African Charter. In EVANS and MURRAY (eds.), Chap. 2, pp. 49-76.

8. FARER, T. (1998) 'The Rise of the Inter-American Human Rights Regime: No Longer a Unicorn, not yet an Ox'. In D. HARRIS and S. LIVINGSTONE (eds.) The InterAmerican System of Human Rights. Oxford: Clarendon Press, Chap. 32.

9. HARRIS, D. (1998) "Regional Protection of Human Rights: The Inter-American Achievement". In D. HARRIS and S. LIVINGSTONE (eds.), The Inter-American System of Human Rights. Oxford: Clarendon Press, Chap.1.

10. HENRY, STEINER J. (1991) Diverse partners: Non-Governmental Organizations in the human rights movement: Harvard Law School Human Rights Program and Human Rights Internet.

11. JUMA, D. (2009) 'Lost (or Found) in Transition? The Anatomy of the New African Court of Justice and Human Rights'. In A. VON BOGDANDY and R. WOLFRUM, (eds.), Max Planck Yearbook of United Nations Law, 13, pp.283-287.

12. KANYIGA, K. (2008) Contradictions in Neoliberalism: Donors, Human Rights NGOs, and Governance in Kenya. InMUTUA MAKAU (ed.) Human Rights NGOs in East Africa: Political and Normative Tensions. Philadelphia: University of Pennsylvania Press, chap. 8, p.183.

13. KAREN A. MINGST and MARGARET P. KARNS (2007) The United Nations in the 21st century. Oxford: Westview Press.

14. MAINA, P. (2008) "Conclusion: Coming of Age: NGOs and State Accountability in East Africa, MAKAU MUTUA (ed.), p. 310.

15. MBELLE N. (2008) "The Role of Non-Governmental Organizations and National Human Rights Institutions at the African Commission". In EVANS AND MURRAY (eds.), p.289.

16. OLOKA-ONYANGO, J. (2008) "NGO Struggle for Economic, Social and Cultural Rights in UTAKE: A Ugandan Perspective, in MAKAU MUTUA (ed.), chap. 4, p. 75. 
Les Organisations Non-Gouvernementales (ONGs) de défense des droits de l'homme au Burundi : promouvoir la revendication judiciaire des droits.

17. RAMCHARAN, G. BERTRAND (1979) The Role of Regional, National and Local Institutions: Future Perspectives, in RAMCHARAN G. BERTRAND (eds.) Human Rights: Thirty Years After The Universal Declaration, chap.10.

18. TOLLEY, H., JR. (1994) The International Commission of Jurists: Global Advocates for Human Rights. Philadelphia: University of Pennsylvania Press.

19. WELCH, C. (1995) Protecting Human Rights in Africa: Roles and Strategies of NonGovernmental Organizations. Philadelphia: University of Pennsylvania Press.

20. VANDENGINSTE, S. (2011) Stones Left Unturned: Law and Transitional Justice in Burundi. Antwerp: Intersentia.

21. VASAK, K. (1982). In K. VASAK and P. ALSTON(eds.) The International Dimensions of Human Rights, Vol. 2, p.451.

IV. Articles de revues

1. ALFORD, WILliAM, P. (2000) "Exporting "the pursuit of happiness", Harvard Law Review, 113: 1677-1715.

2. Alston, P. (1988) "Making Space for New Human Rights: The Case of the Right to Development”, 1 Harvard Human Rights Yearbook, 1, 3, pp. 29-30.

3. ALSTON, P. AND QUINN, G. (May. 1987) "The Nature and Scope of States Parties' Obligations under the International Covenant on Economic, Social and Cultural Rights, Human Rights Quarterly vol. 9, N. 2: pp. 156-229.

4. DONNELLY, J. (2007) 'The Relative Universality of', HumanRightsQuarterly29, pp. 281-306.

5. DONNELLY, J. (1985) "In Search of the Unicorn: The Jurisprudence and the Politics of the Right to Development”, 15 CAL. W. INT'L L. J., pp. 473-482.

6. HENRY, STEINER P. (1998) "Political Participation as a Human Right."Harvard Human Rights Yearbook, pp. 109-110.

7. JUMA, D. (2007) 'Access to the African Court on Human and Peoples' Rights: A Case of the Poacher turned Gamekeeper', Essex Human Rights Review, 4, 2.

8. LEONARD S. RUBENSTEIN (Nov., 2004) "How International Human Rights Organizations Can Advance Economic, Social, and Cultural Rights: A Response to Kenneth Roth", Human Rights Quarterly, Vol. 26, No. 4, pp. 845-865.

9. MARKS, S. (1981) “Emerging Human Rights: A New Generation for the 1980s?”, Rutgers law review, 33, pp. 435-451.

10. MEDINA, C. (1990) The Inter-American Commission on Human Rights and the InterAmerican Court of Human Rights: Reflections on a Joint Venture, Human Rights Quarterly, Vol. 12, 4, pp.439-464.

11. MEI, A.P. van der., (2005) 'The new African Court on Human and Peoples' Rights: Towards an effective human rights protection mechanism for Africa?' (2005) 18 Leiden Journal of International Law, p.113. 
12. MOHAMED, ABDELSALAM A. (1999) 'Individual and NGO Participation in Human Rights Litigation before the African Court of Human and Peoples' Rights: Lessons from the European and Inter-American Courts of Human Rights', Journal of African Law, 43, 2: pp. 201-213.

13. MURRAY, R. (1997) Decisions by the African Commission on Human Rights on individual communications Under the African Charter on Human and Peoples' Rights, 46 International and Comparative Law Quarterly 412, 413.

14. MUTUA, M. (2008) "Human Rights and Powerlessness: Pathologies of Choice and Substance”, Buffalo Law Review, 56, pp. 1025-103.

15. MUTUA, M. (Apr. 2008) "Human rights in Africa: the Limited Promise of Liberalism”, African Studies Review, vol. 51, N. 1: p. 22.

16. MUTUA, M. (2007) Standard Setting in Human Rights: Critique and Prognosis, $\mathrm{Hu}$ man Rights Quarterly, Vol. 29, 3, pp. 547-630.

17. MUTUA, M. (2001) Savages, Victims, and Saviors: The Metaphor of Human Rights, Harvard International Law journal, 42, pp. 201-245.

18. MUTUA, M. (1999) The African Human Rights Court: A Two-Legged Stool? Human Rights Quarterly 21, pp.342-363.

19. MUTUA, M. (1995-1996) The Ideology of human rights, 36 Va. J. Int'l L.: P.589.

20. NTAHIRAJA, B. et NKURUNZIZA, N. (2010) ' L'Etat face aux droits sociaux de ses citoyens: cas du droit à la santé au Burundi. ', Rule of Law Program for Sub-Saharan Africa, Librairie Africaine d'Etudes Juridiques, Vol. 7: p.20-45.

21. ODINKALU, C. ANSELM(2003) Back to the Future: The Imperative of Prioritizing for the Protection of Human Rights in Africa, Journal of African Law, Vol. 47, No. 1, pp. 1-37.

22. ODINKALU, C. ANSELM (May 2001) Analysis of Paralysis or Paralysis by Analysis? Implementing Economic, Social, and Cultural Rights Under the African Charter on Human and Peoples' Rights, Human Rights Quarterly, Vol. 23, 2, pp.327-369.

23. ODINKALU, C. ANSELM AND CHRISTENSEN, C. (May, 1998) The African Commission on Human and Peoples' Rights: The Development of Its Non-State Communication Procedures, Human Rights Quarterly, Vol. 20, No. 2, pp. 235-280.

24. ÖSTERDAHL, I. (1998) 'The jurisdiction ratione materiae of the African Court on Human and Peoples' Rights: A Comparative Critique', Review of the African Commission on Human and Peoples' Rights, 7(2), p. 132.

25. SHEDRACK, C. AGBAKWA†(2002) "Reclaiming Humanity: Economic, Social, and Cultural Rights as the Cornerstone of African Human Rights", Yale Human Rights \& Development L. J., Vol. 5

26. VANDENGINSTE, S. (2008) 'Pouvoir et droit au Burundi : un commentaire (principalement) juridique spécifiquement une analyse sur l'arrêt du 5 juin 2008 de la Cour Constitutionnelle dans l'affaire RCCB 213', Dialogue, № 243, pp. 45-65.

27. VILJOEN, F. '(2004) 'A Human Rights Court for Africa, and Africans,' Brooklyn Journal of International Law 1, 30, pp. 14-22 
28. VILJOEN, F. AND LOUW, L. (Jan., 2007) 'State Compliance with the Recommendations of the African Commission on Human and Peoples' Rights 1994-2004, The American Journal of International Law, 101, 1, pp. 1-34

29. WELCH JR., CLAUDE E., (September 2003) Human Rights NGOs and the Rule of Law in Africa, Journal of Human Rights, vol. 2, 3, pp. 315-327

30. WISEBERG, LAURIE S. (Summer 1994) 'The African Commission on Human and Peoples' Rights', African Studies Association, Issue: A Journal of Opinion, Vol. 22, No. 2: pp. 34-41

31. WOLfGANG, B. (1993) The African Charter and Commission on Human and Peoples' Rights: How to Make it More Effective, 11 Netherland quarterly of Human Rights, 25, 31 .

V. Thèses et mémoires

1. NDAYIKENGURUKIYE, M. (2005) The international human rights law as a source of law in the Burundian judicial system, Master Dissertation, Makerere University, Kampala.

2. NKURUNZIZA, N. (2012) The Potential for, and Limits to an Approach "From Below" to Transitional Justice in the Burundian Context. Master Dissertation, Trinity College Dublin, Dublin.

VII. Jurisprudence et décisions des organes régionaux

\section{Décisions de la Commission Africaine des Droits de l'Homme et des Peuples}

Communication 155/96, Social and Economic Rights Action Center (SERAC) and Center for Economic and Social Rights (CESR) c. Nigéria, CADHP, 15ème Rapport d'activités: $2001-2002$; 